\title{
Represent precipitation-induced geological hazards in Earth system models using artificial intelligence
}

\author{
Zeli Tan $^{1 *}$, Qizhi $\mathrm{He}^{1}$, Qing Zhu², Chang Liao ${ }^{1}$ \\ ${ }^{1}$ Pacific Northwest National Laboratory, Richland, WA, USA; ${ }^{2}$ Lawrence Berkeley National \\ Laboratory, Berkeley, CA, USA; ${ }^{*}$ Correspondence to: Zeli Tan (zeli.tan@pnnl.gov)
}

Focal Area: Predictive modeling through the use of AI techniques and AI-derived model components; the use of AI and other tools to design a prediction system comprising of a hierarchy of models

\section{Science Challenge and Objectives}

Precipitation-induced geological hazards, such as debris flow, landslides, mudflow and rockfalls (hereafter referred to as landslides), pose serious threats to public safety in many areas through the world. As residential properties and infrastructure in the US have increasingly expanded into landslide-prone areas and the drivers of landslides (e.g., wildfires and hurricanes) are predicted to intensify under climate warming, losses and fatalities from landslides are likely to increase in the future. ${ }^{1}$ Although our understanding of geoenvironmental factors and mechanisms contributing to landslides has greatly improved, only moderate progress has been made in predicting landslides out of well-studied watersheds. ${ }^{2}$ Furthermore, explicitly representing various landslide-related processes in Earth system models (ESMs), from the buckling of local bearing elements in granular materials, to frictional sliding between grains, formation of microcracks in the soil matrix, rupture of capillary bridges, or breakage of plant roots ${ }^{3}$ is still very unlikely within the next decade, even with the help of exascale computers.

To advance modeling and prediction of geological hazards under the changes of land use and climate, we propose a paradigm-shift Artificial Intelligence (AI) based method in which both ESM simulated hydrological and ecological conditions and remote-sensing/harmonized land surface data are assimilated for realistic time-varying hillslope-scale landslide prediction. This AI-based hybrid model will enable implicit representation of macropore-scale soil processes and hillslope-scale heterogeneity of soil and vegetation conditions that is impossible for the resolution of ESMs. Our overarching goal is to achieve accurate prediction of landslide occurrences under land use and land cover change (LULCC) and climate change in mountainous regions across the globe, including the disturbance of natural vegetation and increase of extreme weathers. Additionally, we will intend to complement and leverage the work of improving the predictability of extreme precipitation in ESMs using AI.

\section{Background and Significance}

Shallow landslides (generally $<2 \mathrm{~m}$ depth) induced by heavy precipitation and abrupt snowmelt can occur with limited precursory signals and thus represent a ubiquitous and destructive natural hazard in steep mountainous regions. This geological hazard can be fostered by a range of geoenvironmental factors including lithology, soils, land use, and morphological conditions (e.g., slope, aspect, curvature, and drainage area) which can be altered by natural and human activities. ${ }^{4}$ Based on our improved understanding of factors and mechanisms contributing to landslides, various empirically or physically based models have been developed in recent decades to predict its occurrence..$^{5-7}$ 
Empirically based models are usually adopted for the basins where a certain amount of inventoried landslide events is available for the derivation of the empirical rainfall thresholds. However, because rainfall is not the only factor that causes landslides, a certain degree of uncertainty is unavoidable in the definition of rainfall thresholds. ${ }^{2}$ Another drawback of empirically derived thresholds is that they are based on the assumption of stationary hydroclimate conditions and thus cannot predict the evolution of landslide hazards under changing environment, for example, LULCC, large forest fires' occurrence and sediment availability's fluctuation.

In contrast, physically based models use spatially variable characteristics with a simplified dynamic hydrological model to derive threshold values of soil water potential for landslide prediction in which although rainfall is the most important input parameter antecedent soil moisture can also be accounted. ${ }^{2}$ In such models, the hydrological role of vegetation in slope stabilization is represented by reducing the amount of water in the soil and thus increasing the soil matric suction effect. ${ }^{6}$ The mechanical effects of vegetation are represented by the tensile strength of the root network and the frictional properties of the roots which reinforce the soil. ${ }^{6}$ But these models usually require calibration over a well-specified type of landslides and, in general, are difficult to apply over large areas where detailed knowledge of input parameters (e.g., soil thickness, groundwater conditions, shear resistance parameters) is very difficult to acquire. $^{2}$

Due to the weaknesses of empirically and physically based landslide models, landslides have not been successfully represented in ESMs. Furthermore, ESMs will unlikely explicitly represent macropore-scale soil processes contributing to landslide occurrence in the next decade, ${ }^{8}$ from the buckling of local bearing elements in granular materials, to frictional sliding between grains, formation of microcracks in the soil matrix, rupture of capillary bridges, or breakage of plant roots, even with the rapid evolution of computational resources. In addition, in most steep forested mountains where landslide risk is high, the presence of macropores due to connected root structures, biological activity, fractures, large clasts, and lenses leads to preferential and funneled flows that violate the assumptions in the current generation of ESMs' hydrological components.

AI, a type of data-driven machine learning model, has been successfully applied in the prediction of hydrological variables, such as soil moisture, ${ }^{11}$ stream flow, ${ }^{12}$ and other associated phenomena. By encoding governing equations that describe flow and transport physics into AI architectures, the recent study proposed a neural network-based framework for data assimilation, enabling the estimation of hydraulic conductivity and prediction of hydrological variables, simultaneously. ${ }^{13}$ As such, AI could be a potentially effective approach to bring paradigm shift understandings of landslides' response to LULCC and climate change.

\section{Methods}

To achieve accurate prediction of landslide occurrences under LULCC and climate change in mountainous regions across the globe, we propose to build an AI-based model for landslide prediction and integrate this model into DOE's Energy Exascale Earth System Model (E3SM). We envision that this approach is able to fill the gap between the good scientific understanding of landslide's geoenvironmental factors and mechanisms and the extreme difficulty in representing these factors and mechanisms in ESMs. First, although the soil processes contributing to landslides occur at spatial and temporal scales that are unresolved by ESMs, 
hydrological and ecological conditions, for example soil moisture, water table, vegetation distribution and root network, that drive these soil processes are usually explicitly represented by ESMs. Second, although hydrological and ecological conditions are usually only simulated for the whole hillslope or catchment, the gradient of these hydrological and ecological conditions along the hillslope are frequently captured by high-resolution remote sensing or harmonized land surface and soil products, such as soil moisture, vegetation cover, soil depth, soil texture and morphological conditions (e.g., slope, aspect and curvature). ${ }^{9}$

Thus, we will assimilate both E3SM simulated hydrological and ecological conditions and remote sensing/harmonized land surface properties into the AI-based model to calculate processbased Factor of Safety landslide metric. ${ }^{6,10}$ The model parameters will be trained using both a global landslide inventory database (NASA's Global Landslide Catalog) and simulations of high-fidelity macropore-scale process-based landslide models. ${ }^{3,6}$ The E3SM simulated hydrological conditions that will be assimilated include soil moisture, water table and surface/subsurface runoff. The E3SM simulated ecological conditions that will be assimilated include root topography and vegetation biomass. The remote sensing/harmonized land surface properties that will be assimilated include remote sensing soil moisture, leaf area index, land cover and harmonized data product of soil texture, soil depth and hillslope morphology. ${ }^{7,9}$

After trained (Fig. 1), the AI-based landslide model will act as a surrogate model which relates inputs to outputs of interest to our applications. It will be integrated into E3SM to act as a blackbox computation component in domain algorithms to interact with E3SM simulated prognostic hydrological and ecological conditions and other geographic input data. The prediction accuracy of the AI-based model would be measured by comparing our simulations with the simulation of well-validated regional-scale empirical landslide models. ${ }^{1}$

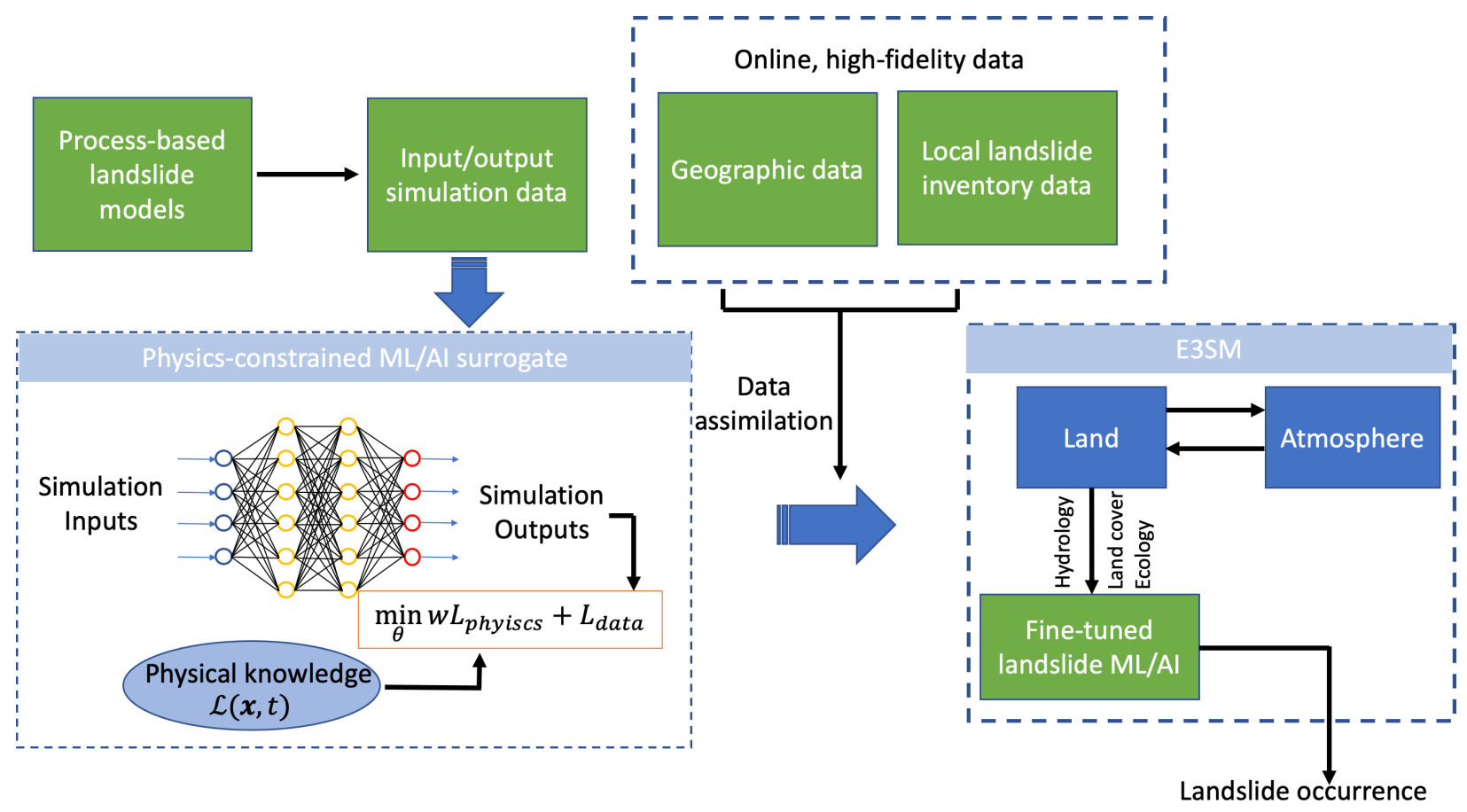

Figure 1. Workflow chart of the AI-based E3SM landslide model. 


\section{References}

${ }^{1}$ Baum, R. L.; Godt, J. W. Landslides 2010, 7, 259-272.

${ }^{2}$ Berti, M.; Martina, M. L. V.; Franceschini, S., et al. J. Geophys. Res. 2012, 117, F04006.

${ }^{3}$ Fan, L.; Lehmann, P.; McArdell, B.; Or, D. Geomorphology 2017, 280, 1-15.

${ }^{4}$ Chang, K. T.; Chiang, S. H.; Chen, Y. C.; Mondini, A. C. Geomorphology 2014, 208, 137-148.

${ }^{5}$ Guzzetti, F.; Peruccacci, S.; Rossi, M.; Stark, C. P. Landslides 2008, 5, 3-17.

${ }^{6}$ Arnone, E.; Caracciolo, D.; Noto, L. V., et al. Water Resour. Res. 2016, 52, 8590-8612.

${ }^{7}$ von Ruette, J.; Lehmann, P.; Or, D. Water Resour. Res. 2013, 49, 6266-6285.

${ }^{8}$ Tan, Z.; Leung, L. R.; Li, H.-Y.; Tesfa, T. J. Adv. Model. Earth Syst. 2018, 10, 2192-2213.

${ }^{9}$ Kirschbaum, D.; Stanley, T. Earth's Future 2018, 6, 505-523.

${ }^{10}$ Posner A. J.; Georgakakos, K. P. Natural Hazards 2015, 79(3), 1825-1845.

${ }^{11}$ Fang, K.; Kifer, D.; Lawson, K.; Shen, C. Water Resour. Res. 2020, 56(12), e2020WR028095.

${ }^{12}$ Shen, C. Water Resour. Res. 2018, 54(11), 8558-8593.

${ }^{13} \mathrm{He}, \mathrm{Q}$; Barajas-Solano, D.; Tartakovsky, G.; Tartakovsky, A. M. Adv. Water Resour. 2020, 141,103610 . 